\title{
Neurotoxin $\beta$-N-methylamino-L-alanine induces endoplasmic reticulum stress-mediated neuronal apoptosis
}

\author{
HAIYING SHEN, KIYOON KIM, YOOJUNG OH, KYUNG SIK YOON, HYUNG HWAN BAIK, \\ SUNG SOO KIM, JOOHUN HA, INSUG KANG and WONCHAE CHOE \\ Department of Biochemistry and Molecular Biology (BK21 Project), \\ Medical Research Center for Bioreaction to Reactive Oxygen Species and Biomedical Science Institute, \\ School of Medicine, Kyung Hee University, Seoul 130-701, Republic of Korea
}

Received September 2, 2015; Accepted September 13, 2016

DOI: $10.3892 / \mathrm{mmr} .2016 .5802$

\begin{abstract}
N-methylamino-L-alanine (BMAA) is a neurotoxin that is closely associated with the incidence of amyotrophic lateral sclerosis, Parkinson's disease and Alzheimer's disease. In cultured neuronal cells, BMAA notably induces the upregulation of endoplasmic reticulum (ER) chaperons and activates the unfolded protein response (UPR) receptor pathways of protein kinase RNA-like endoplasmic reticulum kinase, inositol-requiring kinase 1 and transcription factor 6 . The ER stress-specific protein CCAAT/-enhancer-binding protein homologous protein (CHOP) affords pro-apoptotic responses that cause mitochondrial damage and caspase activation. BMAA also induces the activation of mitogen-activated protein kinase member c-JUN N-terminal kinase, p38 and extracellular signal-regulated kinase, which have been suggested to be involved in the signaling pathway of UPR-mediated apoptosis. Inhibition of ER stress using ER stress antagonist, salubrinal, attenuated the expression of CHOP and alleviated neuronal death. Overexpression of heat shock protein 70 suppressed the activation of UPR receptors and UPR-evoked apoptotic signaling. The present findings demonstrated that ER stress induced by BMAA is the important mediator of neuronal injury and apoptotic death, and suggests development in novel therapeutic strategies for treatment.
\end{abstract}

Correspondence to: Professor Wonchae Choe, Department of Biochemistry and Molecular Biology (BK21 Project), Medical Research Center for Bioreaction to Reactive Oxygen Species and Biomedical Science Institute, School of Medicine, Kyung Hee University, 26 Kyungheedae-ro, Dondaemun-gu, Seoul 130-701, Republic of Korea

E-mail:wchoe@khu.ac.kr

Key words: endoplasmic reticulum stress, apoptosis, $\beta$-N-methylamino-L-alanine, neurodegenerative disease, CHOP, HSP70

\section{Introduction}

$\beta$-N-methylamino-L-alanine (BMAA) is a non-protein amino acid neurotoxin that attracts much attention since it associates with the incidence of neurodegenerative diseases of amyotrophic lateral sclerosis (ALS), Alzheimer's disease (AD) and Parkinson's disease (PD), or combination of them $(1,2)$. BMAA is known to be produced by cyanobacteria (3). By means of biomagnification, BMAA has been identified in aquatic and terrestrial environments (4). A previous study demonstrated that BMAA can be taken up into the brain across the blood-brain barrier via large neutral amino acid carriers, and this intake may be augmented in response to factors, including diet, metabolism, disease and age (5).

The neurotoxicity of BMAA was first concerned with the high incidence of amyotrophic lateral sclerosis/parkinsonism-dementia complex (ALS/PDC), a combination of ALS, PD and dementia (6). Significant levels of BMAA were detected in the brain and spinal cord tissues of patients succumbing to ALS/PDC, whereas no or few quantities of BMAA were detected in those controls (7). A large quantity of in vivo studies have demonstrated memory and behavior disabilities following exposure to BMAA, together with the degeneration and loss of neurons in the central nervous system, and these changes appear proportional to the dose of BMAA administered or the concentration of BMAA present in the nervous tissue $(8,9)$.

Endoplasmic reticulum (ER) stress has been suggested to be involved in human neurodegenerative diseases, including ALS, AD and PD, as well as other disorders (10). Under the condition of stress circumstances, the normal function of the ER is disturbed to induce protein misfolding and aggregation in the lumen of the ER. The ER responds to the accumulation of misfolded protein with the unfolded protein response (UPR) (11). Binding immunoglobulin protein (Bip/Grp78) senses and transfers to the misfolded protein, which makes it dissociate from the ER-transmembrane receptor protein kinase RNA-like endoplasmic reticulum kinase (PERK), inositol-requiring kinase 1 (IRE1) and transcription factor 6 (ATF6), leading to their activation. PERK phosphorylates the alpha subunit of eukaryotic initiation factor 2 (eIF2), which attenuates protein translation. IRE1 $\alpha$ initiates the 
splicing of transcription factor X-box binding protein (XBP1) mRNA, which upregulates the ER chaperon genes, as well as the genes involved in protein degradation. ATF6 $(90 \mathrm{kDa})$ translocates to the Golgi apparatus and is cleaved there to form a $50 \mathrm{kDa}$ transcription factor that translocates to the nucleus, where it activates the transcription of XBP1 and ER chaperon genes. The aim of these responses is to mitigate protein load and restore ER function.

If the disruption to ER is prolonged or aggravated, the UPR develops towards apoptosis. The activation of IRE1 recruits tumor-necrosis factor receptor associated factor 2 (TRAF2) and apoptosis signal-regulating kinase 1 (ASK1), and triggers the c-JUN N-terminal kinase (JNK) signaling pathway. JNK triggers human procaspase-4 (or murine procaspase-12) and downstream caspases (12). The phosphorylation of PERK enhances the translation of activating transcription factor 4 (ATF4) via eIF2 $\alpha$ phosphorylation, which induces the expression of ER stress-specific CCAAT/-enhancer-binding protein homologous protein (CHOP) (13). CHOP downregulates the antiapoptotic mitochondrial B-cell lymphoma (Bcl)-2 family, favoring caspase activation.

Understanding the intracellular pathway of BMAA toxicity is important as it not only renders the target for treating BMAA-induced neurotoxicity, but also facilitates the exploration of the pathogenesis of neurodegenerative disorders. Previous studies have indicated that ER stress is involved in the pathogenesis of ALS, PD and AD, and serves a pivotal role in the processing of neuronal degeneration and death (10). In the present study, the intracellular molecular mechanism of BMAA-induced ER stress and apoptosis was assessed in the cultured HT22 hippocampal cells, Neuro-2a and SH-SY5Y neuroblastoma cells.

\section{Materials and methods}

Materials. BMAA, 3-(4,5-Dimethylthiazol-2-yl)-2,5-diphenyl tetrazolium bromide (MTT), propidium iodide (PI) and salubrinal (Sal) were purchased from Sigma-Aldrich (St. Louis, MO, USA). Thapsigargin (Tg) and tunicamycin (Tm) was obtained from Biomol Research Labs (Plymouth Meeting, PA, USA). Antibodies specific to Bcl-2 (cat. no. sc7382), Bcl-xl (cat. no. sc7195), Bcl-2-associated X protein (Bax; cat. no. sc493), CHOP (cat. no. sc7351), GRP78 cat. no. sc1050), GRP94 (cat. no. sc120647), ATF6 $\alpha$ (p90; cat. no. sc22799), eIF2 $\alpha$ (cat. no. sc133132) and sXBP-1 (cat. no. sc8015) were purchased from Santa Cruz Biotechnology, Inc. (Santa Cruz, CA, USA). Antibodies specific to cleaved JNK (cat. no. 9252), p38 (cat.no. 9212), ERK (cat.no. 9102), caspase-9 (cat.no. 9508), cleaved caspase-3 (cat. no. 9664), poly-ADP ribose polymerase (PARP; cat. no. 9532), phosphorylated (P-)ASK1 (cat. no. 3761), P-eIF2 $\alpha$ (cat. no. 3597), P-JNK (cat. no. 9255), P-p38 (cat. no. 9211) and P-ERK (cat. no. 9101) were purchased from Cell Signaling Technology, Inc. (Danvers, MA, USA). Antibodies against cyclophilin B (CypB; cat. no. ab16045) and protein disulfide isomerase (PDI; cat. no. ab2792) were purchased from Abcam (Cambridge, UK). Antibodies against $\beta$-tubulin (cat. no. sc9104), $\alpha$-actinin (cat. no. sc15336) and actin (cat. no. sc1616) were purchased from Santa Cruz Biotechnology, Inc. GAPDH antibody (cat. no. CSA-335) was purchased from Enzo Life Sciences, Inc. (Farmingdale,
NY, USA). Lipofectamine 2000 was purchased from Gibco (Thermo Fisher Scientific, Inc., Waltham, MA, USA). Small interfering (si)RNAs against control scrambled and CHOP were obtained from Santa Cruz Biotechnology, Inc. Gene Silencer siRNA transfection reagent was purchased from Gene Therapy System (San Diego, CA, USA). DAPI nucleic acid stain was purchased from Molecular Probes (Eugene, OR, USA). Phosphate-buffered saline (PBS), 4\% paraformaldehyde, ethanol, methanol, sodium dodecyl sulfate (SDS), Triton-X-100 and $\mathrm{NaHCO}_{3}$ were purchased from Sigma-Aldrich.

Cell culture. The human neuroblastoma cell line, SH-SY5Y, mouse hippocampal cell line, HT22 and mouse neuroblastoma cell line, Neuro-2a, were purchased from the American Type Culture Collection (Manassas, VA, USA). Cells were cultured in Dulbecco's modified Eagle's medium (DMEM), supplemented with $10 \%(\mathrm{v} / \mathrm{v})$ fetal bovine serum (Hyclone; GE Healthcare Life Sciences, Logan, UT,USA) 100 U/ml penicillin and $100 \mu \mathrm{g} / \mathrm{ml}$ streptomycin (Gibco; Thermo Fisher Scientific, Inc.), in a humidified $5 \%(\mathrm{v} / \mathrm{v}) \mathrm{CO}_{2}$ incubator at $37^{\circ} \mathrm{C}$. For the treatment, BMAA was dissolved in a physiological concentration $(10 \mathrm{mM})$ of $\mathrm{NaHCO}_{3}$, as it has been reported that $\mathrm{HCO}_{3}$ is required for mediating BMAA toxicity $(14,15)$. The cells were serum-starved for $1 \mathrm{~h}$ and incubated with BMAA, as previously described $(14,15)$. On completion of incubation, the cells were washed thoroughly with PBS and subjected to various analyses.

MTT assay. Cell viability was evaluated using the conversion of MTT to MTT-formazan by mitochondrial enzymes. Neuronal cells were seeded into 12 -well plates at a density of $4 \times 10^{5}$ cells/well in growth medium and cultured to $70 \%$ confluence. Following serum starvation for $3 \mathrm{~h}$, the cells were treated with various concentrations of BMAA. After 24 or $48 \mathrm{~h}$ incubation at $37^{\circ} \mathrm{C}$, the cells were washed three times with PBS, and $30 \mu 1$ MTT solution $(5 \mathrm{mg} / \mathrm{ml})$ was added to the cells and subsequently incubated for $1 \mathrm{~h}$ at $37^{\circ} \mathrm{C}$. The medium was removed carefully and $300 \mu \mathrm{l}$ dimethyl sulfoxide was added to dissolve the blue formazan in living cells. Finally, the absorbance at $540 \mathrm{~nm}$ was measured using an ELISA reader (Multiskan EX; Thermo Lab systems, Beverly, MA, USA).

Western blot analysis. For western blot analysis, neuronal cells $\left(3 \times 10^{5}\right.$ cells/well $)$ were seeded for $24 \mathrm{~h}$ into a $6-\mathrm{cm}$ culture dish. The cells were washed twice with ice-cold PBS and total cell lysates were prepared in a lysis buffer containing $50 \mathrm{mM}$ Tris- $\mathrm{HCl}$ (pH 7.4), $150 \mathrm{mM} \mathrm{NaCl}$, $1 \%$ Triton X-100, $50 \mathrm{mM} \mathrm{NaF}, 5 \mathrm{mM}$ sodium pyrophosphate, $1 \mathrm{mM}$ EDTA, $1 \mathrm{mM}$ EGTA, $1 \mathrm{mM}$ DTT, $0.1 \mathrm{mM}$ PMSF and protease inhibitor cocktail. The whole cell lysates were centrifuged $\left(12,000 \times \mathrm{g}\right.$ for $10 \mathrm{~min}$ at $\left.4^{\circ} \mathrm{C}\right)$ for removal of cellular debris. The protein concentration was determined by the Lowry method using a Bio-Rad DC protein assay kit (Bio-Rad Laboratories, Inc., Hercules, CA, USA). Cell lysates containing equal quantities of protein $(50 \mu \mathrm{g})$ were resolved by $8-15 \%$ SDS-polyacrylamide gel electrophoresis and transferred onto nitrocellulose membranes. Each membrane was blocked with a solution containing $5 \%$ non-fat milk in Tris-buffered saline with $0.05 \%$ Tween-20 (TBST) for $1 \mathrm{~h}$ at room temperature. Following blocking, the membranes were 
incubated with the primary antibodies (dilution, 1:2,000) in TBST overnight at $4^{\circ} \mathrm{C}$. Following incubation, the membranes were washed for $1 \mathrm{~h}$ with TBST and were further probed with secondary HRP-conjugated anti-rabbit, anti-mouse or anti-goat immunoglobulin G (dilution, 1:2,000; AbFrontier, Seoul, South Korea) in TBST for $1 \mathrm{~h}$ at room temperature. The immune complexes were visualized using an enhanced chemiluminescence detection system (Pierce; Thermo Fisher Scientific, Inc.), according to the manufacturer's protocol.

SiRNA. siRNA transfections were performed using Lipofectamine 2000 transfection reagent (Thermo Fisher Scientific, Inc.). CHOP siRNA target sequences, purchased from Dharmacon (GE Healthcare Life Sciences, Chalfont, UK) were as follows: Sense, 5'-CUGGGAAACAGCGCA UGA A-3' and antisense, 5'-UUCAUGCGCUGUUUC CCAG-3'. The universal negative control (Dharmacon; GE Healthcare Life Sciences) was used as the scrambled siRNA. HT22 cells were seeded into 6-well plates overnight and transferred into $1 \mathrm{ml}$ serum-free DMEM prior to transfection. Scrambled control and CHOP siRNA were incubated with transfection reagent for $5 \mathrm{~min}$ at room temperature, and the mixtures were subsequently added to the cells. Following $12 \mathrm{~h}$ incubation, $1 \mathrm{ml}$ DMEM containing $20 \%$ calf serum was added to each well. For experiments, the cells were transfected with siRNA for $24 \mathrm{~h}$ and were subsequently exposed to BMAA for $24 \mathrm{~h}$. The efficiency of siRNA-based interference of CHOP was monitored by western blot analysis.

Flow cytometry for DNA content. The cells were seeded at $2 \times 10^{5}$ cells $/ \mathrm{ml}$ density in $100-\mathrm{mm}$ dishes. Following incubation for $24 \mathrm{~h}$, the cells were harvested, washed twice with PBS, and fixed with ice-cold $75 \%$ ethanol at $4^{\circ} \mathrm{C}$ for $24 \mathrm{~h}$. The cells were subsequently pelleted by centrifugation at $1,000 \times \mathrm{g}$ for $5 \mathrm{~min}$ at $4^{\circ} \mathrm{C}$ and the ethanol layer was discarded. Following washing with PBS, the fixed cells were treated with $0.5 \mu \mathrm{g} / \mathrm{ml}$ RNase A in PI buffer for $30 \mathrm{~min}$ at $37^{\circ} \mathrm{C}$. At the end of the treatment, PI $(20 \mu \mathrm{g} / \mathrm{ml})$ was added and the cells were stained for $30 \mathrm{~min}$ in the dark. The cell cycle was then analyzed for DNA content using Kaluza flow cytometry software (Beckman Coulter, Orange County, CA, USA).

Plasmid construction and transfection. Full-length cDNA of Hsp70 was obtained by reverse transcription-polymerase chain reaction (PCR). The primers, synthesized by Macrogen, Inc. (Seoul, South Korea), used for PCR amplification were 5'-CAC CACCTACTCCGACAACCA-3' and 5'-GCCCCTAATCTA CCTCCTCAATG-3'. The PCR reaction mixture $(40 \mu \mathrm{l})$ contained $2 \mathrm{mM} \mathrm{MgCl} 2,0.2 \mathrm{mM}$ dNTP, $1 \mu \mathrm{M}$ primers and 1 unit Taq DNA polymerase. The samples were amplified using 30 cycles of $60 \mathrm{sec}$ denaturation at $94^{\circ} \mathrm{C}$ and $30 \mathrm{sec}$ annealing at $62^{\circ} \mathrm{C}$. The product was subsequently cloned into pcDNA3.1-HA plasmid (Invitrogen; Thermo Fisher Scientific, Inc.) using BamHI and KpnI enzymes. The plasmids were transfected into cells with Lipofectamine 2000 reagent, according to the manufacturer's protocol. The cells were treated with BMAA $24 \mathrm{~h}$ after transfection. The efficiency of transfection was monitored by western blot analysis with anti-HA antibody (cat. no. sc138; Santa Cruz Biotechnology, Inc.).
DAPI staining and confocal microscopy. Equal numbers of neuronal cells were seeded onto glass coverslips in a 12-well microplate. Following harvesting, the cells were washed in PBS and fixed in $4 \%$ paraformaldehyde containing $0.25 \%$ Triton $\mathrm{X}-100$ for $10 \mathrm{~min}$ at $22^{\circ} \mathrm{C}$. Cell nuclei were stained with $6.5 \mu \mathrm{g} / \mathrm{ml}$ DAPI, $16 \%$ polyvinyl alcohol and $40 \%$ glycerol $\mathrm{C}$ for $30 \mathrm{~min}$ at $37^{\circ} \mathrm{C}$. The cells on the glass coverslips were covered with histological mounting medium (National Diagnostics, Atlanta, GA, USA). Fluorescence was evaluated in three independent high-power fields (magnification, x400) using an LSM510 confocal laser microscope (Carl Zeiss, Oberkochen, Germany). The excitation/emission wavelengths for DAPI was 358/461 nm. Apoptotic cells were identified with nuclear morphology of apoptosis, involving chromatin condensation and DNA fragmentation.

Statistical analyses. The results were expressed as the mean \pm standard deviation from at least three independent experiments. Statistical analyses were performed using Student's t-test. Unless indicated otherwise, $\mathrm{P}<0.05$ was considered to indicate a statistically significant difference.

\section{Results}

BMAA induces apoptotic neuronal death in a dose- and time-dependent manner. Previous studies have demonstrated that the induction of neuronal degeneration and death, rather than of excitotoxicity or oxidative stress, requires high concentrations of BMAA ( $\geq 1.0-3.0 \mathrm{mM})$ (14-16). The present study first estimated the neurotoxic effect of BMAA on the viability of neuronal cells using an MTT reduction assay. HT22, Neuro-2a and SH-SY5Y cells were treated with increasing doses of BMAA (1.0, 2.0 and $3.0 \mathrm{mM})$. At $24 \mathrm{~h}$ after the beginning of exposure to BMAA, no significant injury occurred with BMAA concentrations $<1.0 \mathrm{mM}$, whereas a mild cell death occurred at the $1.0 \mathrm{mM}$ level, a moderate cell death occurred at $2.0 \mathrm{mM}$ and a marked cell death occurred at $3.0 \mathrm{mM}$ (Fig. 1A). Next, to detect cell injury in the different stages, cell viability was measured at 12,24 and $48 \mathrm{~h}$, and the results revealed a time-dependent increase in cell death (Fig. 1B).

In response to $\mathrm{BMAA}$ exposure, the expression of anti-apoptotic Bcl-2 and Bcl-xL revealed much lessened levels compared with the control; however, the level of pro-apoptotic Bax was notably increased, which shifted the Bax/Bcl-2 ratio to withdraw the survival signal and favor apoptosis in a dose-dependent manner. Cytochrome $c$ is released by the regulation of the Bcl-2 family proteins and binds to pro-caspase-9, which cleaves initiator caspase-9 and in turn cleaves the effector caspase-3. The cleavage of poly (ADP-ribose) polymerase (PARP) is also involved in DNA repair and programmed cell death. The cleavage of the caspases and PARP was detected in BMAA-treated cells, however, not in control cells (Fig. 1C). Collectively, these data indicated that BMAA treatment induces apoptotic neuronal injury and death in a dose- and time-dependant manner.

Activation of UPR signaling and UPR signaling-evoked apoptotic pathway. In response to BMAA exposure, the phosphorylation of PERK and eIF2 $\alpha$, and the splicing of XBP1 were increased in a BMAA dose-dependent manner. By contrast 
to the increased level of the above proteins, the expression of full length ATF6 (ATF6 p90) was detected with decreasing levels in the cytosol, suggesting the cleavage and translocation of ATF6 into the nucleus (Fig. 2A). ER chaperons have been demonstrated as monitors of ER stress. The predominant ER chaperon proteins involve Bip/Grp78, protein disulfide isomerase, Grp94 and Cyp B (17-19). The much increased levels of the ER chaperons Bip/Grp78, Grp94, PDI, and CypB were represented in BMAA-treated cells compared with control (Fig. 2B), suggesting the result in response to the accumulation of misfolding proteins in the ER. The persistent ER stress initiates UPR signaling-mediated pro-apoptotic signaling. The phosphorylated ASK1 and CHOP were detected in BMAA-treated cells in a dose-dependent manner, however, not in the control cells, suggesting the activation of ER stress-mediated apoptotic pathway in response to BMAA (Fig. 2C).

In order to confirm the pro-apoptotic action of $\mathrm{CHOP}$, the present study performed RNA interference experiments using siRNA to reduce the levels of CHOP. HT22 and Neuro-2a cells were transfected with CHOP siRNA, followed by BMAA treatment. Western blot analysis revealed that the cells transfected with CHOP siRNA exhibit enhanced expression of $\mathrm{Bcl}-2$, whereas this reduces the expression of cleaved-PARP compared with those untreated, suggesting that the inhibition of ER-specific CHOP pathway inhibits pro-apoptotic signaling (Fig. 2D). To further evaluate DNA fragmentation in each neuronal cell, the percentage of subG1 cells was analyzed via flow cytometry as a measure of apoptotic index. Cells transfected with CHOP siRNA exhibited a reduced population with cell cycle arrest in the sub-G1 phase compared with those without transfection or transfected with control scrambled siRNA (Fig. 2E). Taken together, these findings notably indicate that the UPR signaling proteins and UPR-mediated apoptotic pathway are activated as a result of ER stress in BMAA-treated neuronal cells.

Activation of the ERK, JNK and p38 MAPK pathway. Extracellular signal-regulated kinase (ERK), JNK and p38 mitogen-activated protein kinase (MAPK) are the three major members of the MAPK family. These MAPK members have been suggested to be involved in the signaling pathway of UPR-mediated apoptosis (20). Activated IRE1 recruits TRAF2 to trigger the JNK-induced apoptotic signaling pathway (12). IRE1 also leads to apoptosis by stimulating the phosphorylation of ERK and p38 MAPK during ER stress (21). CHOP is also post-translationally regulated upon phosphorylation on serine residues 78 and 81 by p38 MAPK, which increases its activity and confers p38 as a substrate of ASK1 along the IRE1-ASK1-p38 pathway (22). Western blot analysis revealed that the phosphorylation of ERK, JNK and p38 MAPK were markedly activated in BMAA-treated neurons (Fig. 3A). The findings revealed that ERK, JNK and p38 MAPK are involved in the UPR-evoked apoptotic pathway to contribute to the induction of neuronal death.

Sal is a selective inhibitor of phosphatase complexes that dephosphorylate eIF- $2 \alpha$ and has been primarily used to investigate ER stress-induced apoptosis (23). Neuronal cells were pretreated with $20 \mu \mathrm{M}$ Sal for $30 \mathrm{~min}$. As a positive control, cells were exposed to UPR inducers, Tg (an inhibitor of Sarco-ER Ca2+ ATPase pump) and Tm (an inhibitor of N-linked

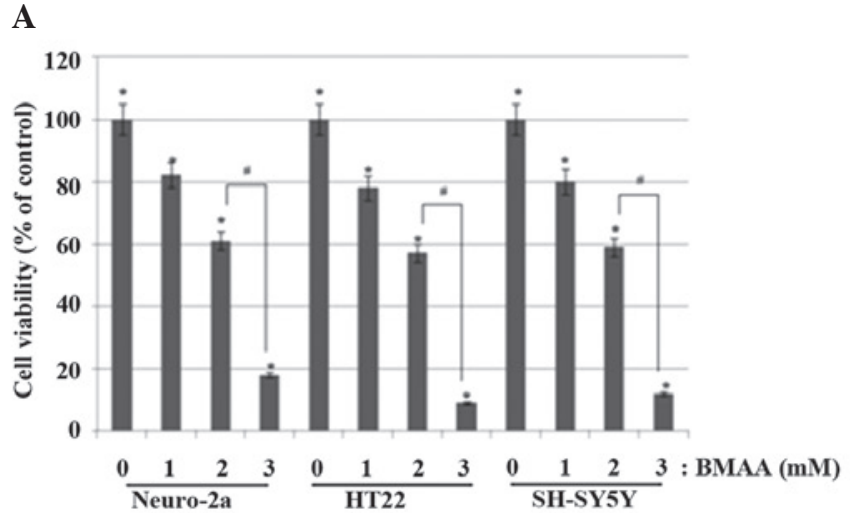

B
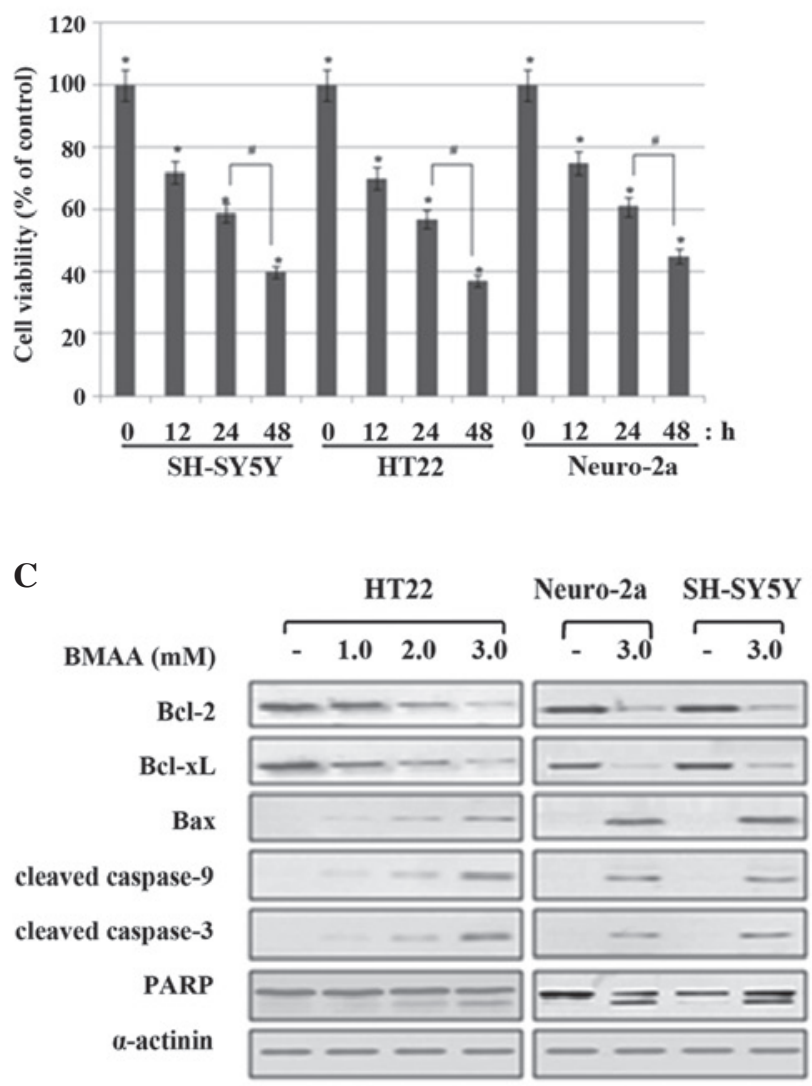

Figure 1. Effects of BMAA on cell viability and apoptosis of neuronal cells. (A) Neuronal cells were treated with $1.0,2.0$, or $3.0 \mathrm{mM}$ BMAA and were analyzed at $24 \mathrm{~h}$ after the beginning of exposure to BMAA. The percentage cell viabilities are representative of at least three different experiments and are expressed as the mean \pm standard deviation ( ${ }^{*} \mathrm{P}<0.05$ vs. untreated cells; ${ }^{\text {"}} \mathrm{P}<0.05$ vs. $2.0 \mathrm{mM}$-treated cells). (B) The cell viabilities were measured at 12 , 24 and $48 \mathrm{~h}$ after treatment with $2.0 \mathrm{mM} \mathrm{BMAA}$. The data are expressed as the mean \pm standard deviation obtained from at least three independent experiments ( $\mathrm{P}<0.05$ vs. untreated cells; ${ }^{*} \mathrm{P}<0.05$ vs. 24 h-treated cells). (C) The cells were treated with increasing doses of BMAA $(1.0,2.0$ and $3.0 \mathrm{mM})$ for $24 \mathrm{~h}$. The protein expression levels of Bcl-2 family members, and the cleavage of caspase and PARP, were assessed by western blot analysis. $\alpha$-actinin was used as a loading control. The data are representative of at least three different experiments. BMAA, $\beta$-N-methylamino-L-alanine; Bcl, $\mathrm{B}$-cell lymphoma; Bax, $\mathrm{Bcl}$-2-associated $\mathrm{X}$ protein; PARP, poly-ADP ribose polymerase.

glycosylation). BMAA-induced ER stress was significantly inhibited by Sal treatment, as assessed by the phosphorylation of eIF $2 \alpha$, JNK and p38 MAPK, and protein expression levels 
A

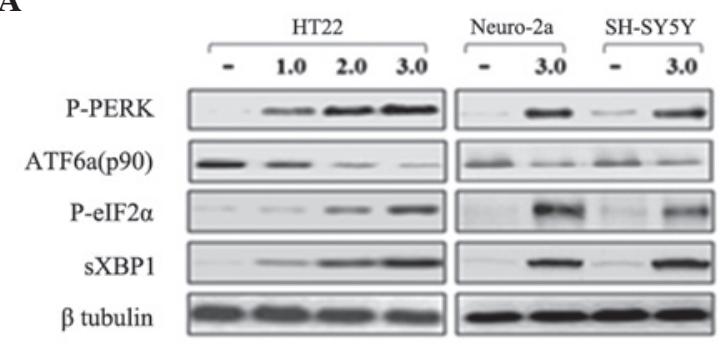

C

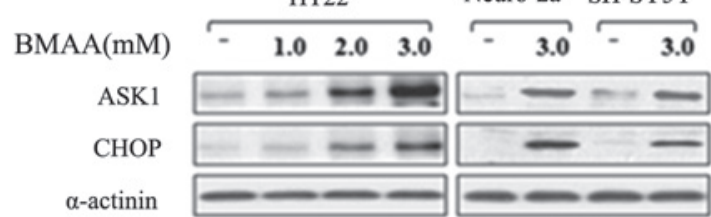

B

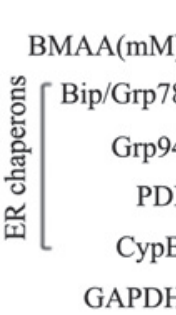

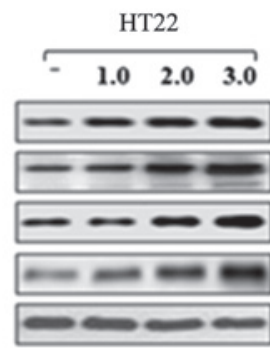

Neuro-2a SH-SY5Y
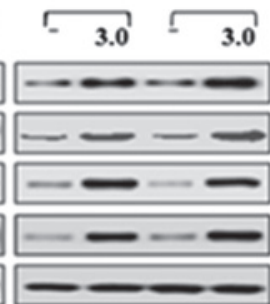

D

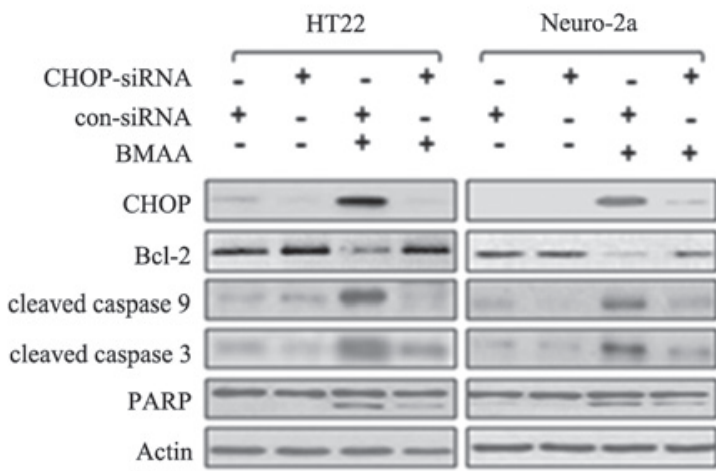

$\mathbf{E}$

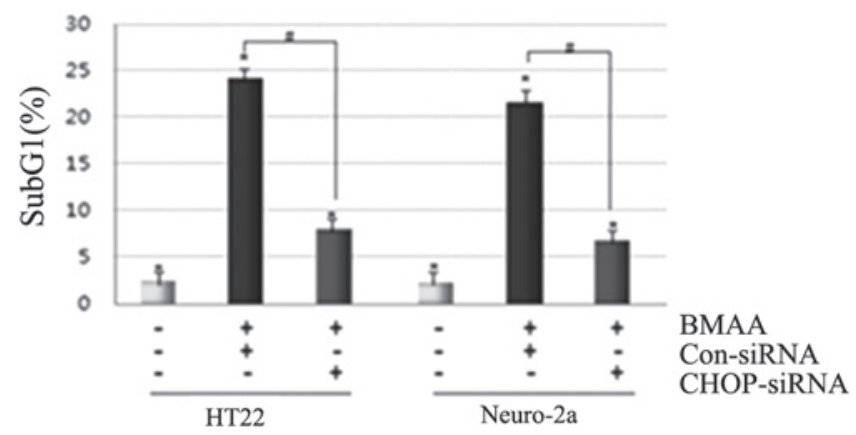

Figure 2. Treating BMAA induces the activation of UPR signaling and the UPR signaling-evoked apoptotic pathway. Neuronal cells were exposed to increasing concentrations of BMAA and detected at $24 \mathrm{~h}$. Western blot analysis was performed with antibodies specific to (A) P-PERK and eIF2 $\alpha$, spliced XBP1, IRE10 and full length ATF6 $\alpha$, (B) ER chaperon, and (C) ASK1 and CHOP. (D) Neuronal cells were transfected with con-siRNA and CHOP-siRNA for $24 \mathrm{~h}$ and were subsequently treated with $3.0 \mathrm{mM}$ BMAA for $24 \mathrm{~h}$. Knockdown efficiency of CHOP-siRNA, the protein expression levels of the Bcl-2 family, the cleaved caspases and PARP were assessed by western blot analysis. $\beta$-tubulin, GAPDH $\alpha$-actinin or actin were used as a loading control. The results are representative of at least three independent experiments. (E) The cells were transfected with con-siRNA and CHOP-siRNA, and the percentage of subG1 cells was determined by flow cytometry. The data are expressed as the mean \pm standard deviation from at least three independent experiments (" $\mathrm{P}<0.05$ vs. untreated cells; ${ }^{\#} \mathrm{P}<0.05$ vs. BMAA-treated cells transfected with con-siRNA). BMAA, $\beta$-N-methylamino-L-alanine; Bcl, B-cell lymphoma; Bax, Bcl-2-associated X protein; PARP, poly-ADP ribose polymerase; P-, phosphorylated; PERK, protein kinase RNA-like endoplasmic reticulum kinase; ATF, transcription factor 6; XBP, $\mathrm{X}$-box binding protein; ER, endoplasmic reticulum; PDI, protein disulfide isomerase; CypB, cyclophilin B; ASK, apoptosis signal-regulating kinase; CHOP, CCAAT/-enhancer-binding protein homologous protein; con, control; si, small interfering; eIF2, eukaryotic initiation factor 2.

of Bip/Grp78, GRP94 and CHOP. Similar suppression effect of Sal also occurred in ER stress induced by Tg and Tm (Fig. 3B). Pretreatment of Sal notably increases neuronal survival, as monitored by MTT assay, while the cell death rate appears to remain higher compared with the control (Fig. 3C). These findings indicated that suppressing ER stress can effectively restrain apoptotic neuronal death induced by BMAA treatment and facilitate cell survival with protective effect.

Overexpression of Hsp70 suppresses UPR-evoked apoptotic signaling. Molecular chaperons have been demonstrated to serve the role of correcting misfolded protein and preventing them from aggregation (24). A previous study suggested that Hsp70 takes multiple protective effects on ER stress and stress-induced apoptosis (25). Neuronal cells were treated with
BMAA following transfection with Hsp70. We first confirmed the overexpression of Hsp70 by western blot assay. Under the condition of Hsp70 overexpression, the phosphorylation of IRE1 and PERK, and the cleavage of ATF6 were significantly attenuated compared with the cells without transfection. The expression of ER chaperon GRP94 and PDI were lowered in Hsp70 transfected cells compared with non-transfected cells. Bcl-2 and Bcl-xL exhibited an increased level, while CHOP and Bim/Bax, and cleavage of caspases and PARP were at lower levels in Hsp70-transfected cells (Fig. 4A). Hsp70 transfection markedly increases neuronal survival, as measured using an MTT reduction assay, and the defensive role of HSP70 is not limited to cell type (Fig. 4B). The present study subsequently examined the protective effect using confocal microscopy. As visualized by DAPI nuclear staining, Hsp70 protected neuronal 
cells against nuclear condensation and fragmentation induced by BMAA, when compared with those without transfection (Fig. 4C). To further evaluate DNA fragmentation in each neuronal cell, flow cytometry was performed as an apoptotic index. Cells transfected with HSP70 represented a lower population of cell cycle arrest in the sub-G1 phase, indicating that HSP70 effectively inhibits BMAA-induced apoptosis (Fig. 4D). Taken together, these data clearly demonstrated that HSP70 overexpression suppresses the activation of UPR sensors and UPR-evoked apoptotic signaling as a protective effect.

\section{Discussion}

An important sign of neurodegenerative diseases is the accumulation and aggregation of misfolded proteins (26). Recent studies suggested that BMAA is incorporated into proteins by mischarged transfer RNA synthetase (tRNAs), and this misincorporation causes protein misfolding and aggregation in neuronal cells (27). A previous study also showed that BMAA can be assembled into protein chains via binding to serine transfer RNA, resulting in improper folding of the protein (28). These findings were consistent with the fact that a large fraction of BMAA was detected from protein precipitates in ALS, PD and AD samples.

Previous studies also implicated that the excitotoxicity of BMAA is mediated by the activation of $\alpha$-amino-3-hydroxy-5-isoxazolepropionic acid (AMPA) receptors (29), N-methyl-D-aspartate (NMDA) receptors and metabotropic glutamate receptors (mGluR) (30). This activation causes the increase of intracellular calcium levels (31), and results in reactive oxygen species (ROS) production and oxidative stress state (32). Misfolded protein accumulation, glutamate-induced excitotoxicity, calcium dyshomeostasis and oxidative stress are involved in the factors that induce ER stress (33).

ER is the important organelle that facilitates protein folding and an accumulation of misfolded proteins in the lumen of the ER is activated in response to ER stress (34). The hallmark of this response is the transcriptional upregulation of ER chaperone proteins. The present study detected increased expression of the ER chaperon protein Bip/Grp78, Grp94, PDI and CypB in response to BMAA-exposure. These ER-resident chaperons and folding enzymes serve multiple roles in promoting protein folding and endoplasmic reticulum-associated protein degradation (ERAD). Their upregulation indicates the disturbance of protein folding and the accumulation of misfolded proteins in the ER. The present results revealed the phosphorylation of PERK and eIF2 $\alpha$, the activation of IRE1, and the splicing of XBP1, as well as the reduction of ATF6 p90. The response of the three UPR pathways aims to mitigate the overload of misfolded protein in the ER. It indicates that BMAA treatment causes the dysfunction of ER and the aggregation of misfolded proteins in the ER.

CHOP is activated to initiate the ER stress-induced apoptotic response of cells, which amplifies the pro-apoptotic signal by altering the balance between $\mathrm{Bcl}-2$ and $\mathrm{Bax}$ (35). The expression of CHOP was markedly induced in response to BMAA treatment. The role of CHOP was confirmed by siRNA-mediated knockdown of the protein, which significantly diminished apoptosis. This suggested that CHOP serves a critical role in
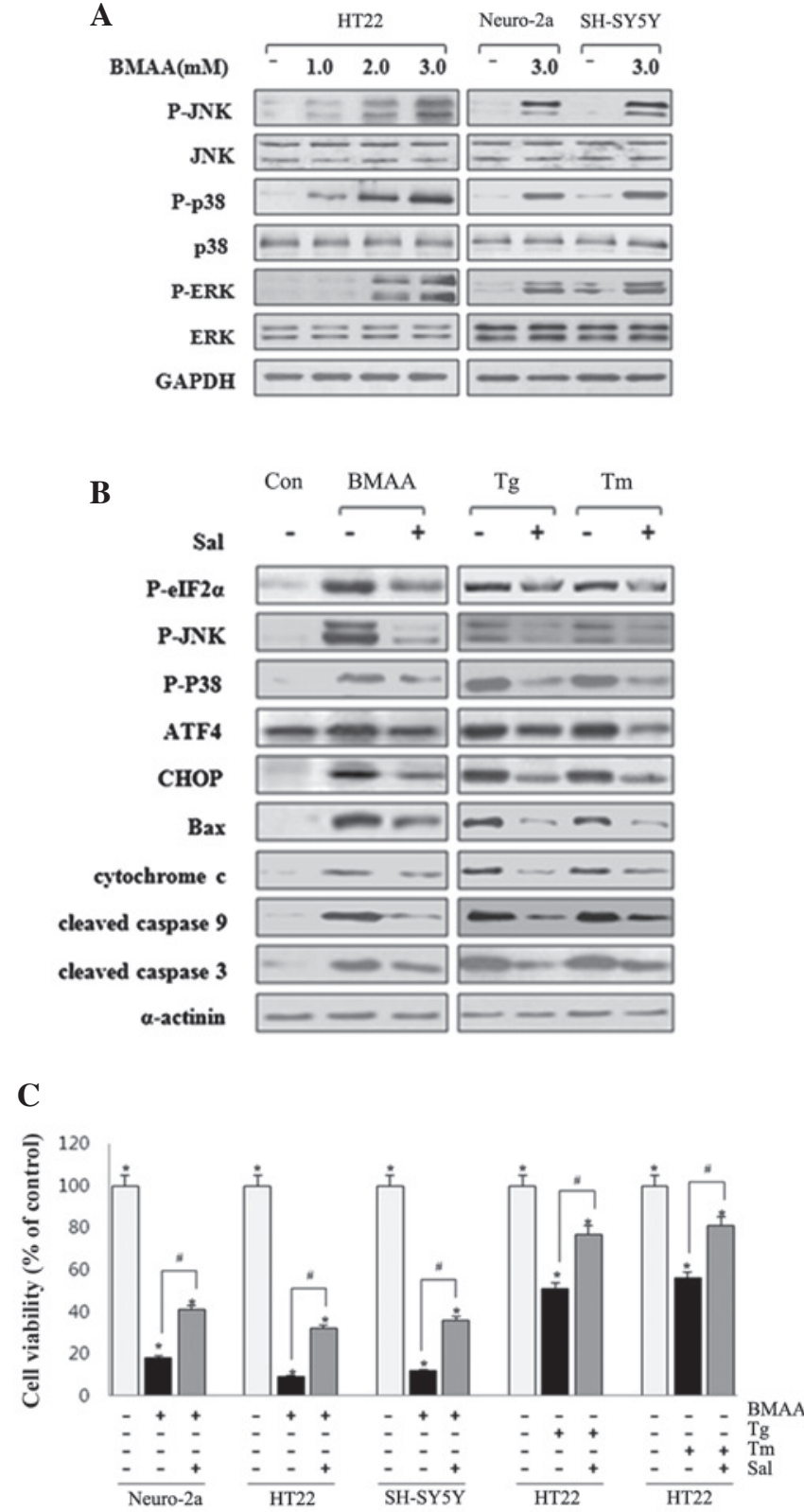

Figure 3. BMAA initiates ER stress-induced phosphorylation of p38, JNK, and ERK. (A) HT22, Neuro-2a and SH-SY5Y cells were treated with BMAA at $1.0,2.0$ or $3.0 \mathrm{mM}$ for $24 \mathrm{~h}$. The protein expression levels of P- and total p38, JNK and ERK were measured by western blotting. (B) The cells were pretreated with $20 \mu \mathrm{M}$ Sal for $30 \mathrm{~min}$, followed by $3.0 \mathrm{mM}$ BMAA, $1 \mu \mathrm{M} \mathrm{Tg}$ or $10 \mu \mathrm{g} / \mathrm{ml} \mathrm{Tm}$ for $24 \mathrm{~h}$, respectively. Sal inhibits ER stress-induced phosphorylation of eIF2 $\alpha$, p38, and JNK, and the expression levels of CHOP, Bax and cleaved caspases. The results shown are representative of at least three independent experiments. (C) Cell viability was measured using an MTT assay under the above conditions. The data are expressed as the mean \pm standard deviation obtained from at least five independent experiments ( ${ }^{*} \mathrm{P}<0.05$ vs. untreated cells. ${ }^{\#} \mathrm{P}<0.05$ vs. Sal-treated cells). BMAA, $\beta$-N-methylamino-L-alanine; Bax, B-cell lymphoma-2-associated X protein; P-, phosphorylated; ATF, transcription factor 6; ER, endoplasmic reticulum; CHOP, CCAAT/-enhancer-binding protein homologous protein; JNK, c-Jun $\mathrm{N}$-terminal kinases; ERK, extracellular signal-regulated kinase; Sal, salubrinal; Tg, thapsigargin; Tm, tunicamycin.

BMAA-induced cell death. Previous studies demonstrated that the MAPKs are activated by ER stress inducers and are involved in ER stress-induced apoptosis (20). Activated JNK and p38, which mainly contribute to stress-induced apoptosis, are suggested serve a role of increasing the expression and 
B
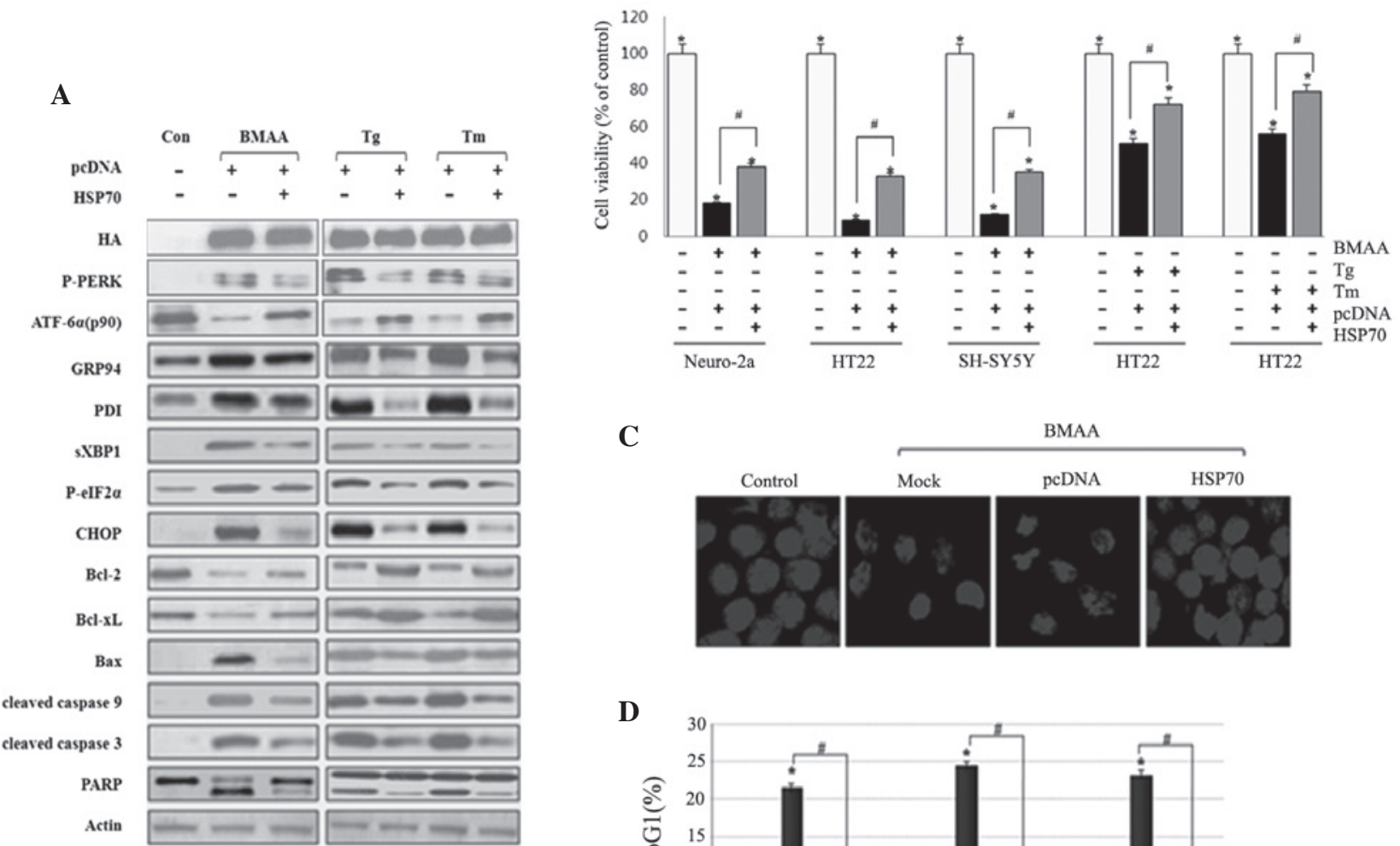

C

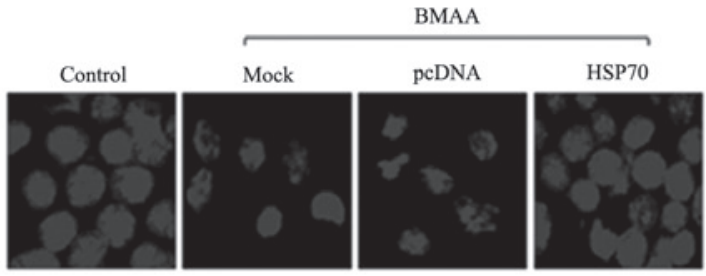

D

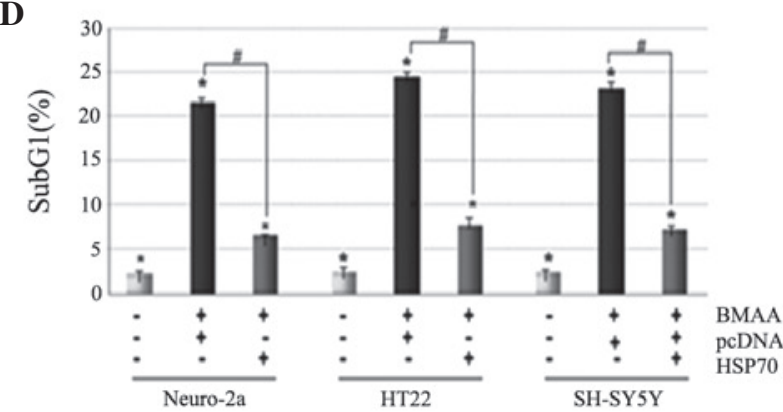

Figure 4. Overexpression of HSP70 suppresses ER stress-mediated neuronal death induced by BMAA, Tg and Tm. Following transfection with DNA constructs of pcDNA and HSP70 for $24 \mathrm{~h}$, the cells were treated with $3.0 \mathrm{mM} \mathrm{BMAA}, 1 \mu \mathrm{M} \mathrm{Tg}$, or $10 \mu \mathrm{g} / \mathrm{ml} \mathrm{Tm}$ for $24 \mathrm{~h}$. Con cells are untreated cells. (A) Western blotting for ER stress and apoptosis markers was performed. HSP70 was tagged with HA and the expression of HSP70 in the indicated transfectants was analyzed by western blotting. Consistent results are representative of at least three different experiments. (B) Cell viability was measured using an MTT assay The percentage viability was plotted as the mean \pm standard deviation of at least five experiments $\left({ }^{*} \mathrm{P}<0.05\right.$ vs. untreated cells; ${ }^{*} \mathrm{P}<0.05$ vs. BMAA-treated cells transfected with pcDNA only). (C) Nuclear morphology was visualized by DAPI nuclear staining. Mock are untransfected cells and con are untreated cells. The experiments were performed in triplicate and visualized under a confocal microscope. (D) The percentage of subG1 cells was analyzed via flow cytometry. The data are expressed as the mean \pm standard deviation obtained from at least three independent experiments $\left({ }^{*} \mathrm{P}<0.05\right.$ vs. untreated cells; ${ }^{*} \mathrm{P}<0.05$ vs. BMAA-treated cells transfected with pcDNA only). BMAA, $\beta$-N-methylamino-L-alanine; Bcl, B-cell lymphoma; Bax, Bcl-2-associated X protein; P-, phosphorylated; ATF, transcription factor 6; ER, endoplasmic reticulum; CHOP, CCAAT/-enhancer-binding protein homologous protein; Tg, thapsigargin; Tm, tunicamycin; HSP, heat shock protein; HA, hemagglutinin; XBP, X-box binding protein; PARP, poly-ADP ribose polymerase; PDI, protein disulfide isomerase; PERK, protein kinase RNA-like endoplasmic reticulum kinase; con, control; eIF2, eukaryotic initiation factor 2.

transcriptional activity of CHOP (35). The phosphorylation of ERK, JNK and p38 MAPK was observed, indicating the intense cellular stress caused by BMAA-exposure.

Sal has been reported to protect against neuronal injury in the rat brain through inhibiting ER stress, and Sal is capable of penetrating into brain tissue in vivo (36). Sal, which inhibits eIF2a dephosphorylation, alleviates the phosphorylation of JNK and p38 MAPK, and the translation of ATF4, thus suppressing the expression of CHOP. Pretreatment with Sal alleviates BMAA-induced ER stress and expression of CHOP, and enhances neuronal survival. Hsp70 prevents the misfolded proteins from aggregation, and allows them to refold (37). Hsp70 also affects the apoptotic pathway at the levels of cytochrome $c$ release and caspase activation, and protects cells against stress-induced apoptosis (25). Overexpression of Hsp70 reduces the expression of ER chaperons and UPR receptors, suggesting that the initial UPR signaling is suppressed. The pro-apoptotic signaling proteins, CHOP and Bax, are downregulated, and the cleaved caspase- 9 and -3 are attenuated, indicating that ER stress-mediated apoptotic cell death is inhibited. These findings indicated that ER stress is a highly promising therapeutic target for BMAA-induced neuronal injury and death, and implicated ER stress inhibitors and molecular chaperons as potential pharmacological agents.

The present findings demonstrated that BMAA induces ER stress-mediated apoptosis in the cultured neuronal cells. BMAA induces the upregulation of ER chaperons and the activation of UPR receptors of PERK, IRE1 and ATF6, as well as the phosphorylation of MAPK member JNK, p38 and ERK. The ER stress-specific protein CHOP is activated and drives pro-apoptotic responses that causes mitochondrial damage and caspase activation. Furthermore, the present study demonstrated that inhibition of ER stress using ER stress antiagonist 
Sal and Hsp70 protein mitigates neuronal damage and apoptosis as a protective effect.

\section{Acknowledgements}

The present study was supported by a grant from the Kyung Hee University in 2012 (no. KHU-20121733) and a grant from the Basic Science Research Program through the National Research Foundation of Korea, which is funded by the Ministry of Education (no. NRF-2013R1A1A2060694).

\section{References}

1. Murch SJ, Cox PA, Banack SA, Steele JC and Sacks OW: Occurrence of beta-methylamino-l-alanine (BMAA) in ALS/PDC patients from Guam. Acta Neurol Scand 110: 267-269, 2004.

2. Pablo J, Banack SA, Cox PA, Johnson TE, Papapetropoulos S, Bradley WG, Buck A and Mash DC: Cyanobacterial neurotoxin BMAA in ALS and Alzheimer's disease. Acta Neurol Scand 120: 216-225, 2009

3. Banack SA, Johnson HE, Cheng R and Cox PA: Production of the neurotoxin BMAA by a marine cyanobacterium. Mar Drugs 5: 180-196, 2007.

4. Banack SA and Cox PA: Biomagnification of cycad neurotoxins in flying foxes: Implications for ALS-PDC in Guam. Neurology 61: 387-389, 2003.

5. Smith QR, Nagura H, Takada Y and Duncan MW: Facilitated transport of the neurotoxin, beta-N-methylamino-L-alanine, across the blood-brain barrier. J Neurochem 58: 1330-1337, 1992.

6. Plato CC, Cruz MT and Kurland LT: Amyotrophic lateral sclerosis-Parkinsonism dementia complex of Guam: Further genetic investigations. Am J Hum Genet 21: 133-141, 1969.

7. Spencer PS, Palmer VS, Herman A and Asmedi A: Cycad use and motor neurone disease in Irian Jaya. Lancet 2: 1273-1274, 1987.

8. Karamyan VT and Speth RC: Animal models of BMAA neurotoxicity: A critical review. Life Sci 82: 233-246, 2008.

9. Holtcamp W: The emerging science of BMAA: Do cyanobacteria contribute to neurodegenerative disease? Environ Health Perspect 120: A110-A116, 2012.

10. Lindholm D, Wootz H and Korhonen L: ER stress and neurodegenerative diseases. Cell Death Differ 13: 385-392, 2006.

11. Rasheva VI and Domingos PM: Cellular responses to endoplasmic reticulum stress and apoptosis. Apoptosis 14: 996-1007, 2009.

12. Urano F, Wang X, Bertolotti A, Zhang Y, Chung P, Harding HP and Ron D: Coupling of stress in the ER to activation of JNK protein kinases by transmembrane protein kinase IRE1. Science 287: 664-666, 2000.

13. Boyce M and Yuan J: Cellular response to endoplasmic reticulum stress: A matter of life or death. Cell Death Differ 13: 363-373, 2006.

14. Weiss JH and Choi DW: Beta-N-methylamino-L-alanine neurotoxicity: Requirement for bicarbonate as a cofactor. Science 241: 973-975, 1988.

15. Lobner D, Piana PM, Salous AK and Peoples RW: Beta-N-methylamino-L-alanine enhances neurotoxicity through multiple mechanisms. Neurobiol Dis 25: 360-366, 2007.

16. Okle O, Stemmer K, Deschl U and Dietrich DR: L-BMAA induced ER stress and enhanced caspase 12 cleavage in human neuroblastoma SH-SY5Y cells at low nonexcitotoxic concentrations. Toxicol Sci 131: 217-224, 2013.

17. Lee AS: The ER chaperone and signaling regulator GRP78/BiP as a monitor of endoplasmic reticulum stress. Methods 35: 373-381, 2005.
18. Kim I, Xu W and Reed JC: Cell death and endoplasmic reticulum stress: Disease relevance and therapeutic opportunities. Nat Rev Drug Discov 7: 1013-1030, 2008.

19. Walker AK, Farg MA, Bye CR, McLean CA, Horne MK and Atkin JD: Protein disulphide isomerase protects against protein aggregation and is $\mathrm{S}$-nitrosylated in amyotrophic lateral sclerosis. Brain 133: 105-116, 2010.

20. Nagai H, Noguchi T, Takeda K and Ichijo H: Pathophysiological roles of ASK1-MAP kinase signaling pathways. J Biochem Mol Biol 40: 1-6, 2007.

21. Hung JH, Su IJ, Lei HY, Wang HC, Lin WC, Chang WT, Huang W, Chang WC, Chang YS, Chen CC and Lai MD: Endoplasmic reticulum stress stimulates the expression of cyclooxygenase-2 through activation of NF-kappaB and pp38 mitogen-activated protein kinase. J Biol Chem 279: 46384-46392, 2004.

22. Wang XZ and Ron D: Stress-induced phosphorylation and activation of the transcription factor CHOP (GADD153) by p38 MAP Kinase. Science 272: 1347-1349, 1996.

23. Boyce M, Bryant KF, Jousse C, Long K, Harding HP, Scheuner D, Kaufman RJ, Ma D, Coen DM, Ron D and Yuan J: A selective inhibitor of eIF2alpha dephosphorylation protects cells from ER stress. Science 307: 935-939, 2005.

24. Kim YE, Hipp MS, Bracher A, Hayer-Hartl M and Hartl FU: Molecular chaperone functions in protein folding and proteostasis. Annu Rev Biochem 82: 323-355, 2013.

25. Mosser DD, Caron AW, Bourget L, Meriin AB, Sherman MY, Morimoto RI and Massie B: The chaperone function of hsp70 is required for protection against stress-induced apoptosis. Mol Cell Biol 20: 7146-7159, 2000.

26. Ross CA and Poirier MA: Protein aggregation and neurodegenerative disease. Nat Med 10 (Suppl): S10-S17, 2004.

27. Lee JW, Beebe K, Nangle LA, Jang J, Longo-Guess CM, Cook SA, Davisson MT, Sundberg JP, Schimmel P and Ackerman SL: Editing-defective tRNA synthetase causes protein misfolding and neurodegeneration. Nature 443: 50-55, 2006.

28. Dunlop RA, Cox PA, Banack SA and Rodgers KJ: The non-protein amino acid BMAA is misincorporated into human proteins in place of L-serine causing protein misfolding and aggregation. PLoS One 8: e75376, 2013.

29. Rao SD, Banack SA, Cox PA and Weiss JH: BMAA selectively injures motor neurons via AMPA/kainate receptor activation. Exp Neurol 201: 244-252, 2006.

30. Cucchiaroni ML, Viscomi MT, Bernardi G, Molinari M, Guatteo E and Mercuri NB: Metabotropic glutamate receptor 1 mediates the electrophysiological and toxic actions of the cycad derivative beta-N-Methylamino-L-alanine on substantia nigra pars compacta DAergic neurons. J Neurosci 30: 5176-5188, 2010

31. Brownson DM, Mabry TJ and Leslie SW: The cycad neurotoxic amino acid, beta-N-methylamino-L-alanine (BMAA), elevates intracellular calcium levels in dissociated rat brain cells. J Ethnopharmacol 82: 159-167, 2002

32. Liu X, Rush T, Zapata J and Lobner D: beta-N-methylamino-1alanine induces oxidative stress and glutamate release through action on system Xc(-). Exp Neurol 217: 429-433, 2009.

33. Görlach A, Klappa P and Kietzmann T: The endoplasmic reticulum: Folding, calcium homeostasis, signaling and redox control. Antioxid Redox Signal 8: 1391-1418, 2006.

34. Doyle KM, Kennedy D, Gorman AM, Gupta S, Healy SJ and Samali A: Unfolded proteins and endoplasmic reticulum stress in neurodegenerative disorders. J Cell Mol Med 15: 2025-2039, 2011.

35. Oyadomari S and Mori M: Roles of CHOP/GADD153 in endoplasmic reticulum stress. Cell Death Differ 11: 381-389, 2004.

36. Sokka AL, Putkonen N, Mudo G, Pryazhnikov E, Reijonen S, Khiroug L, Belluardo N, Lindholm D and Korhonen L: Endoplasmic reticulum stress inhibition protects against excitotoxic neuronal injury in the rat brain. J Neurosci 27: 901-908, 2007.

37. Chaudhuri TK and Paul S: Protein-misfolding diseases and chaperone-based therapeutic approaches. FEBS J 273: 1331-1349, 2006. 